\title{
Practical three color live cell imaging by widefield microscopy
}

\author{
Jianrun Xia ${ }^{1}$, Song Hon H. Kim ${ }^{1}$, Susan Macmillan ${ }^{1}$ and Ray Truant ${ }^{1 *}$
}

\begin{abstract}
${ }^{1}$ Department of Biochemistry and Biomedical Sciences, McMaster University, HSC 4H24A 1200 Main Street West, Hamilton, Ontario, L8N 3Z5, Canada.
*Corresponding Author: Ray Truant, Department of Biochemistry and Biomedical Sciences, McMaster University, HSC 4H24A 1200 Main Street West, Hamilton, Ontario, Canada, L8N 3Z5. Phone: 01-905-525-9140 ext. 22450; Fax: 01-905-522-9033; Email: truantr@mcmaster.ca
\end{abstract}

Submitted: April 10, 2006; Revised: June 13, 2006; Accepted: June 13, 2006.

Indexing terms: Green Fluorescent Proteins; Blue Fluorescent Protein, Aequorea Victoria.

\section{ABSTRACT}

Live cell fluorescence microscopy using fluorescent protein tags derived from jellyfish and coral species has been a successful tool to image proteins and dynamics in many species. Multi-colored aequorea fluorescent protein (AFP) derivatives allow investigators to observe multiple proteins simultaneously, but overlapping spectral properties sometimes require the use of sophisticated and expensive microscopes. Here, we show that the aequorea coerulescens fluorescent protein derivative, PS-CFP2 has excellent practical properties as a blue fluorophore that are distinct from green or red fluorescent proteins and can be imaged with standard filter sets on a widefield microscope. We also find that by widefield illumination in live cells, that PS-CFP2 is very photostable. When fused to proteins that form concentrated puncta in either the cytoplasm or nucleus, PSCFP2 fusions do not artifactually interact with other AFP fusion proteins, even at very high levels of over-expression. PSCFP2 is therefore a good blue fluorophore for distinct three color imaging along with eGFP and mRFP using a relatively simple and inexpensive microscope.

\section{INTRODUCTION}

Fluorescent protein technology using aequorea victoria fluorescent protein (AFP) derivatives has become an established and essential tool for the cell biology laboratory (1). Enhanced green fluorescent protein (eGFP) has been mutated to enhanced cyan (eCFP) and yellow (eYFP) fluorophores (2), to allow the simultaneous observation of two protein fusions in live cells for co-localization studies and to use the biophysical properties of these fluorophores to detect protein-protein interactions and conformational changes by FRET and FRET-FLIM $(3,4)$. The discovery and development of discosoma species fluorescent protein variants (DFPs) (5), such as monomeric Red Fluorescent Protein (mRFP) (6) and others (7), have led to bright, monomeric, photostable fluorophores that can be distinctly imaged together with eGFP for two color imaging, or with eCFP and eYFP for three color imaging (8). The practical use of these fluorophores for three or four color live cell imaging has a few challenges: the potential for artifactual signals due to eCFP-eYFP DNA recombination from the very similar cDNA sequences in yeast or by plasmid transfection (9), the relatively low photostability of eYFP or BFP $(10,11)$; signal bleeding between eCFP and eYFP channels due to overlapping emission/excitation spectra; and the relatively low amount of fluorescent signal from eCFP or mCerulean blue (12) when using restrictive filters to co-image with eYFP; and the use of sophisticated and expensive microscopes. Some of these problems can be addressed with time-intensive silent mutagenesis of the eCFP/eYFP cDNA to minimize recombination events (9). Signal bleed across filter sets, especially for proteins that inherently form concentrated puncta, is a common artifact in cell biology live cell imaging that can be addressed with the use of sophisticated, but expensive, acoustic-optical tunable filters (13), beam splitters (14) or optical gating technology (15), together with multiple lasers on confocal microscopes that allow spectral distinction with minimal intensity loss. Alternatively, these problems may be 
addressed by less costly methods on a typical widefield fluorescent microscope with minimal expression times, more restrictive bandpass filter technology, and the use of multiple fluorescent proteins that are more spectrally distinct from each other.

To address these practical issues, we investigated whether we could use blue-green derived fluorophores from a third species, aequorea coerulescens, derived from the wild-type green fluorescent protein, aceGFP (16), as a fluorophore to practically co-image with eGFP and mRFP by the method of fluorescent widefield microscopy using a xenon light source. AceGFP has been derived into a photo-activatible monomeric cyan fluorescent protein, or PS-CFP2 (17). We found that this fluorophore had biophysical properties as a blue fluorophore that allowed it to be co-imaged with eGFP and mRFP without any artifactual interactions, minimal photobleaching or spectral bleeding. PSCFP2 appears to be a good fluorophore choice for co-imaging with eGFP or mRFP in live cells on a simple fluorescent microscope with common filter sets.

\section{METHODS}

\section{Plasmid constructs and transfection}

Plasmid expressing eGFP-ASf1 was a kind gift of D.Spector (Cold Spring harbor). EGFP, eCFP, eYFP plasmids (all C1 based) were purchased from Clontech/Invitrogen. MRFP cDNA was a kind gift from R. Tsien (UC Berkely). Huntingtin Q138 exon1 fragment mRFP was made similarly as previously described (18). PSCFP2-Mito and eGFP-mito were made by using Age1 and Xba1 to remove dsRed cDNA from dsRED2-mito (Clontech), and ligating in PSCFP2 with the same restriction endonuclease overhanging ends from PSCFP2-C1 from Evrogen (Moscow, Russia) or eGFP-C1 (Clontech/Invitrogen). PSCFP2-NXF1 was constructed into PSCFP2-C1 similarly as previously described (19). MCerulean Blue-profilin1 was constructed by PCR amplification of human profilin $1 \mathrm{cDNA}$ from a cDNA library with introduced BamH1-EcoR1 ends and ligation into mCerulean Blue-C1 (Kind Gift of D. Piston). EYFPSynapsin1 was constructed by PCR amplification of human synapsin 1 cDNA from a cDNA library with introduced BamH1-EcoR1 ends and ligation into eYFPC1 (Clontech/Invitrogen). All plasmids were transfected using ExGen 500 polyethylimine (Fermentas) (to avoid lipid-based transfection reagent artifactual fluorescence (20)), in $25 \mathrm{~mm}$ culture dishes with glass-coverslip bottoms, constructed as previously described (21). Plasmid were transfected into NIH 3T3 mouse epithelial cells at 500ng each and allowed to express for 24 hours. Cells were grown and imaged at $37 \mathrm{C}, 5 \% \mathrm{CO}_{2}$ in D-MEM media.

\section{Live cell microscopy}

Emission/excitaton filters were in place by the use of filter wheels (Lambda LS, Sutter Instruments, Novato, CA) or commercially available filter set cubes (Brightline, Semrock Corp., Rochester, NY, or Chroma Technologies, Rockingham, VT), on an inverted fluorescent imaging microscope (Nikon Te200, Nikon Canada, Mississauga ON, Hamamatsu ORCA ER camera, Quorum Technologies, Guelph, Ontario, Canada). Light source was a Sutter $175 \mathrm{~W}$ xenon ozonefree lamp through a liquid light guide (Sutter Instruments, Novato, CA), or a $50 \mathrm{~W}$ mercury lap (Hamamatsu). Cells were imaged over time in Bioptechs Delta $\mathrm{T}$ heated tissue culture plates with $5 \% \mathrm{CO}_{2}$ inlet for buffering.

\section{Photostability assays}

NIH 3 T3 cells expressing 500ng of transiently transfected fluorophore-producing plasmids were imaged at 24 hours post transfection using filter sets optimized for each fluorophore (all Semrock sets). Cells were continuously irradiated without shuttering for 300 seconds, and images were captured every 10 seconds. Fluorescence intensity was quantified by pixel intensity using Image J software (NIH), and data was normalized to percent start fluorescence for each fluorophore and plotted over time.

\section{RESULTS AND DISCUSSION}

We visualized PSCFP2 (from PS-CFP2-C1,Evrogen, Moscow, Russia) protein transiently expressed in NIH3T3 cells with a variety of filter sets. In the absence of photoactivation, we could not detect any PSCFP2 fluorescence above autofluorescent background using a commercially available filter sets (Semrock CFP-2432A, or Chroma 31044v2). PSCFP2 can photactivate, or photoconvert, from a blue fluorescent emission to a cyan 
(green-shifted) emission upon irradiation with very intense $405 \mathrm{~nm}$ laser excitation. This fluorophore has emission and excitation maxima of $402 / 468 \mathrm{~nm}$ which was compatible with commercially available DAPI/Hoechst filter sets (Semrock DAPI 5060B, or Chroma 31000v2). PS-CFP2 provided bright violet-blue fluorescence that could be used together with eGFP and mRFP for live cell three-color imaging without signal bleed across filter sets (Semrock DAPI 5060b, GFP3035b, TXRed 40404b), even at long exposure times ( $>1$ second/channel) with very high over-expression of fusion proteins (See Fig. 1a-d).
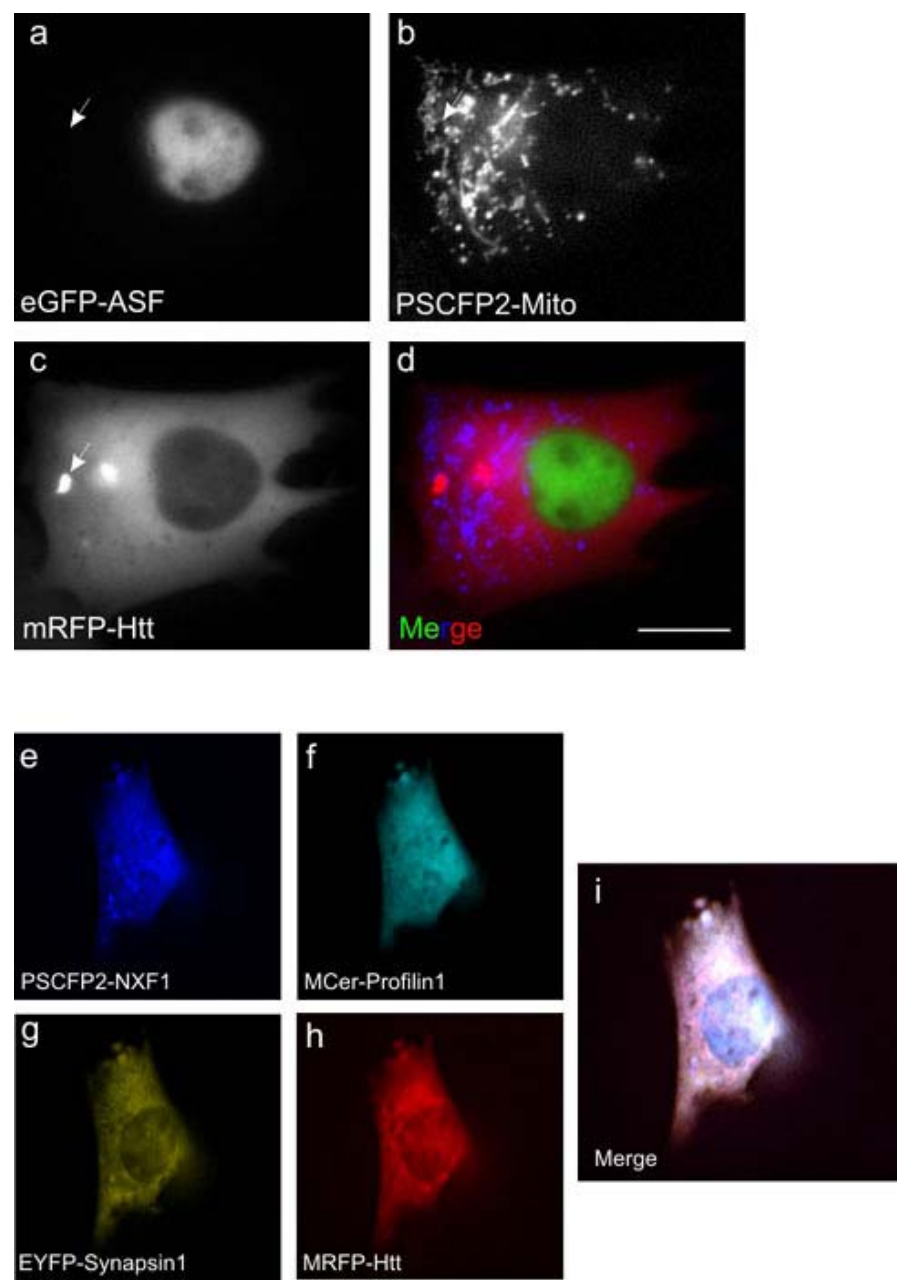

Fig. 1: PSCFP2 Can be Distinctly Imaged with a DAPI/hoechst Filter Set. Panels a-d, Transient transfection of 1ug each eGFP-ASF, PSCFP2-Mito localization signal, mRFP-huntingtin Exon1 Q138 fragment after 24 hours in NIH3T3 (non-plasmid replicating) cells. Less than $200 \mathrm{msec}$ required for each channel of exposure. Scale bar is 10 um. Panels e-l, live cell four color imaging of PSCFP2-NXF1, mCerProfilin1, eYFP-Synapsin1, and mRFP-huntingtin exon1Q138. Distinct signals could be detected for each of the proteins. 4 channel color images and merge were assembled in Imaris 4.3 (Bitplane, Zurich, Switzerland). Scale bar is $10 \mu \mathrm{m}$.

This blue fluorophore allowed the practical observation of three colors in live cells due to the relatively bright signal of each of the three fluorophores $(>200 \mathrm{msec}$ exposure each channel). With mRFP and eGFP, PSCFP2 fusion protein imaging produced no detectable signal bleed, even at 10X time exposure in other channels and with fusions forming concentrated, bright puncta (see Fig. 1 a-d). We found that PSCFP2 could be imaged using filter sets with mCerulean blue (Semrock CFP-2432A), eYFP (Semrock YFP-2427A) and mRFP (Semrock TXRed 40404b) for four color live cell fluorescent imaging (see Fig. 1, panels e-i).

One of the challenges in cell biology imaging is the coobservation of proteins that form bright puncta, causing signal bleeding into other channels. We co-expressed huntingtin exon1 Q138, an aggregation-prone fragment of huntingtin protein (18), as a mRFP fusion protein, eGFP-mito, localized to mitochondria, and PSCFP2NXF1, an mRNA nuclear export factor (19), together in NIH $3 \mathrm{~T} 3$ mouse cells for 24 hours. In each case, the expressed proteins formed distinct, concentrated puncta in either the cytoplasm or the nucleus that could maximize the channel pixel values with $200 \mathrm{msec}$ of exposure (TIFF grayscale value of 255 in each channel) (see Fig. 2A). Despite these bright puncta, no signal bleed was observed between the channels using these standard filter sets.

Another application of fluorescent protein fusions is the long term time course of imaging of multiple proteins. To test how well PSCFP2 would work in this application, we observed the expression of PS-CFP2 NXF1, an mRNA export factor (19) , eGFP-ASF/SF2, a mRNA processing factor located in nuclear puncta, or "speckles" (22), and huntingtinQ138 exon1-mRFP, a fragment of polyglutamine expanded huntingtin protein that is prone to aggregation (18). Using a triple-band dichroic mirror (Semrock, FF436/514/604 Di01) we could distinctly observe NXF1 localization, ASF speckle dynamics and huntingtin aggregation into large, extremely bright inclusions that neither bled signal into the other observed channels nor led to artifactual interaction and recruitment due to aggregation (See Fig. 2B and supplemental video 1). Thus, PSCFP2 does not get recruited into inclusions formed by other fluorescent proteins in vivo. As with other fusion proteins in Figure $2 \mathrm{~A}$, during these long term live-cell observations, we could not detect any bleed of signal between the blue and green channels, indicating no detectable photoconversion of PSCF2 to its activated cyan state. 
A

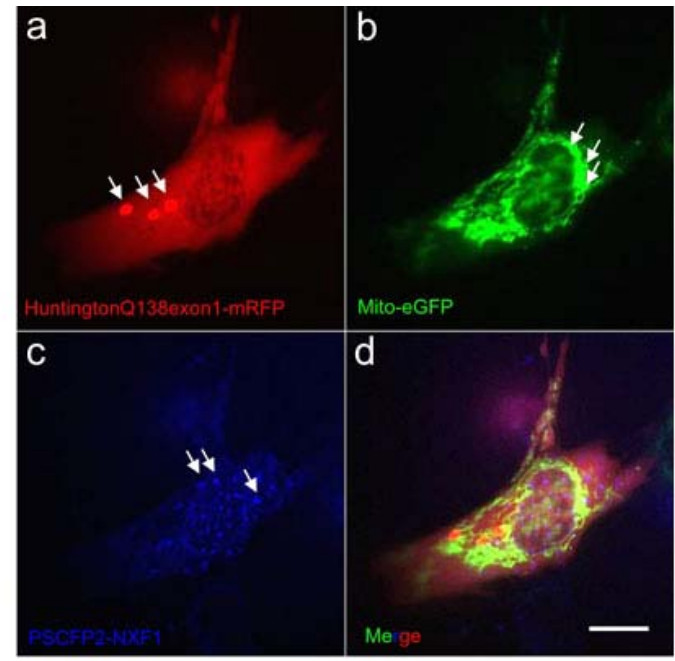

B
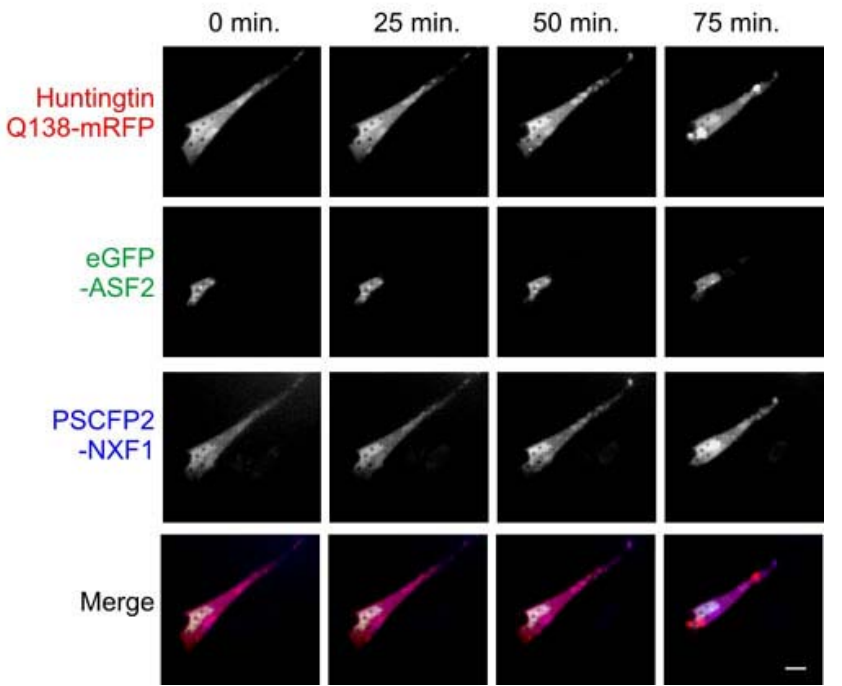

Fig. 2: Live cell Imaging over Time of RGB Fluorescent Proteins That Can form Bright Puncta. A. Three color imaging using a triple cube filter set for DAPI/FITC/Texas Red (Semrock DA/FI/TX-B) and switching only excitation filters for three proteins that form independent bright puncta: huntingtinExon1Q138-mRFP, Mito-eGFP, and PSCFP2-NXf1. Bright puncta in each channels are highlighted with white arrows. B. Time course of mRFP-huntingtin Q138 exon1 fragment, prone to aggregation, eGFPASF2, nuclear speckle protein, and PSCFP2-NXF1, a mRNA export factor in STHdh cells. Three color images were captured every minute for 75 minutes. Developing polyglutamine inclusions of huntingtin can be seen in the red channel without bleed to green or blue channels and no artifactual accumulation of the other two fusion proteins into the large polyglutamine aggregates (top row). Scale bar is $10 \mu \mathrm{m}$. Also in supplemental video 1.

We then tested the practical photo-stability of PS-CFP2 under 175W xenon light (ozone-free, Sutter Instruments, Novato, CA) irradiation in live cells compared to mCeruleanFP, eGFP, eYFP and mRFP (Fig. 3A).

Cells were each transfected with 500ng plasmid per $25 \mathrm{~mm}$ culture dish and observed at the same time posttransfection.

Xia et al. - Practical three color live cell imaging by widefield microscopy www.biologicalprocedures.com
A

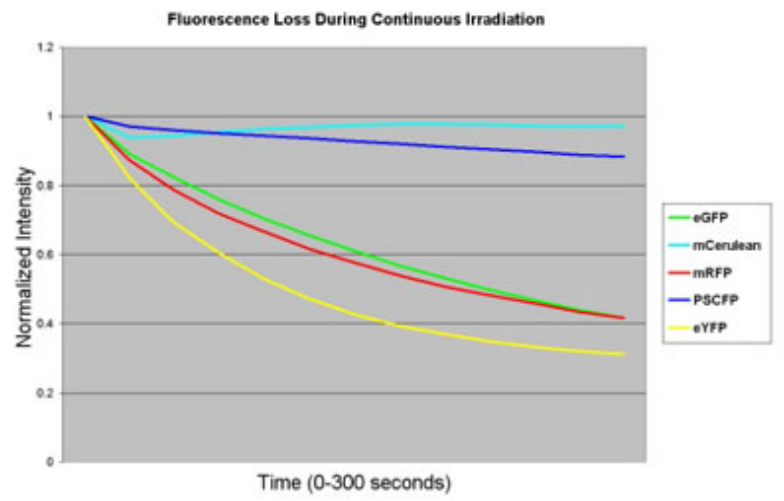

B

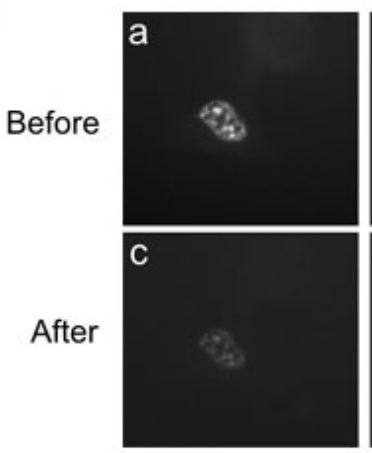

DAPI/Hoechst
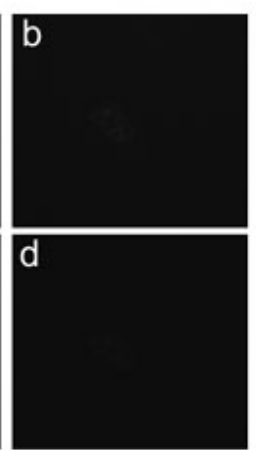

Cyan

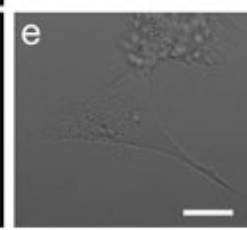

DIC
Fig. 3: Under Wide Field Illumination, PSCFP2 is Photostable. A fluorescent proteins, without fused sequences, were continuously irradiated for 300 seconds with appropriate filter sets, with images captured every two seconds in live NIH 3T3 cells. Monochrome images were quantified using Image $J(\mathrm{NIH})$, normalized to start fluorescent levels, and plotted over time. Deviations across three experiments for each fluorophore was $<5 \%$. B. PSCFP2 does not photoactivate with $175 \mathrm{~W}$ unfiltered xenon light over 15 minutes constant irradiation. Light was provided without filtration through a liquid light guide and using a plan apochromat NA1.3 63X oil immersion objective (Nikon). While slight photobleaching was noted, (panels a versus $c$ ), no cyan fluorescence was detected above CCD camera noise (panels b,d). Scale bar is $10 \mu \mathrm{m}$.

Cells were continuously irradiated and images captured every 10 seconds over a 300 second time course. When intensity measurements were normalized and plotted, we could see that PS-CFP2 and mCerulean blue (an eCFP variant) (12), were the most practically photostable of the fluorophores tested in live cells, with little loss of signal even after 300 seconds of continuous irradiation (equivalent to $\sim 1500$ captured frames), this was in contrast to observed photostability from eYFP and mRFP (see Fig. 3A). EYFP and mRFP photobleaching could be significantly reduced by addition of a neutral density filter (data not shown), taking advantage of the higher quantum yields of these two proteins (6).

PS-CFP2 has been described as a fluorophore that can photo-activate to produce cyan fluorescence upon 
intense 405nm laser irradiation (17). However, using either unfiltered $175 \mathrm{~W}$ xenon light or 50W mercury lamp illumination continuously for as long as 15 minutes, no activation to cyan could be seen, indicating that for even very long-term studies using filtered light and widefield microscopy, the blue fluorophore was stable and distinct from the cyan emission spectrum and inadvertent photoactivation was not a evident (see Fig. 3B). Lack of photoactivation in these experiments was likely due to the weaker light intensity than a brighter, point-focused $405 \mathrm{~nm}$ laser, and by the use of an ozone-free xenon lamp bulb (PE175BF, Perkin Elmer), not producing light at wavelengths below 340nm.

PSCFP2 is a commercially available fluorophore with well-described properties of fluorescence activation using a $405 \mathrm{~nm}$ laser as well as being monomeric, nontoxic to mammalian cells, and can be fused at either its carboxyl- or amino-terminus (17). Here, we report the additional observations that PSCFP2 can be used as a practical and stable fluorophore together with eGFP and mRFP on a relatively simple and inexpensive inverted fluorescent microscope. Practical applications for expression in any species include three or four color imaging of proteins in living cells or very long term studies of single protein expression by 4 dimensional $(\mathrm{x}, \mathrm{y}, \mathrm{z}$, time$)$ analysis, a technique requiring a photostable fluorophore. Due to the voilet-blue fluorescence of PSCFP2, this fluorophore should be compatible for practical $5+$ color observations with far red mRFP variants such as mGrape and mPlum $(7,23)$.

\section{SUPPLEMENTAL INFORMATION}

Supplemental videos of PSCFP2, mRFP and eGFP imaging can be seen online at http://www.science.mcmaster.ca/biochem/faculty/truant/ RGB75min.mov.

\section{ACKNOWLEDGMENTS}

This work is supported by an operating grant and a New Scientist scholarship to RT from the Canadian Institutes of Health Research. We would like to thank the generous and prompt gifts of fluorescent protein plasmids from R. Tsien, D. Piston and R. Campbell. The authors have no conflicts of interest to declare related to this publication.

\section{REFERENCES}

1. Tsien RY. The green fluorescent protein. Annu Rev Biochem 1998; 67:509-544.

2. Llopis J, Westin S, Ricote M, Wang Z, Cho CY, Kurokawa $\mathrm{R}$, et al. Ligand-dependent interactions of coactivators steroid receptor coactivator-1 and peroxisome proliferator-activated receptor binding protein with nuclear hormone receptors can be imaged in live cells and are required for transcription. Proc Natl Acad Sci USA 2000; 97(8):4363-4368.

3. Voss TC, Demarco IA, Day RN. Quantitative imaging of protein interactions in the cell nucleus. Biotechniques 2005; 38(3):413-424.

4. Day RN, Schaufele F. Imaging molecular interactions in living cells. Mol Endocrinol 2005; 19(7):1675-1686.

5. Baird GS, Zacharias DA, Tsien RY. Biochemistry, mutagenesis, and oligomerization of DsRed, a red fluorescent protein from coral. Proc Natl Acad Sci USA 2000; 97(22):11984-11989.

6. Campbell RE, Tour O, Palmer AE, Steinbach PA, Baird GS, Zacharias DA, et al. A monomeric red fluorescent protein. Proc Natl Acad Sci USA 2002; 99(12):7877-7882.

7. Shaner NC, Campbell RE, Steinbach PA, Giepmans BN, Palmer AE, Tsien RY. Improved monomeric red, orange and yellow fluorescent proteins derived from Discosoma sp. red fluorescent protein. Nat Biotechnol 2004; 22(12):1567-1572.

8. He L, Wu X, Simone J, Hewgill D, Lipsky PE. Determination of tumor necrosis factor receptorassociated factor trimerization in living cells by CFP$>$ YFP->mRFP FRET detected by flow cytometry. Nucleic Acids Res 2005; 33(6):e61.

9. Szymczak AL, Workman CJ, Wang Y, Vignali KM, Dilioglou S, Vanin EF, et al. Correction of multi-gene deficiency in vivo using a single 'self-cleaving' 2A peptide-based retroviral vector. Nat Biotechnol 2004; 22(5):589-594.

10. Yang TT, Sinai P, Green G, Kitts PA, Chen YT, Lybarger $\mathrm{L}$, et al. Improved fluorescence and dual color detection with enhanced blue and green variants of the green fluorescent protein. J Biol Chem 1998; 273(14):8212-8216.

11. Griesbeck O, Baird GS, Campbell RE, Zacharias DA, Tsien RY. Reducing the environmental sensitivity of yellow fluorescent protein. Mechanism and 
applications. J Biol Chem 2001; 276(31):29188-29194.

12. Rizzo MA, Springer GH, Granada B, Piston DW. An improved cyan fluorescent protein variant useful for FRET. Nat Biotechnol 2004; 22(4):445-449.

13. Wachman ES, Niu W, Farkas DL. AOTF microscope for imaging with increased speed and spectral versatility. Biophys J 1997; 73(3):1215-1222.

14. Zucker RM, Price O. Evaluation of confocal microscopy system performance. Cytometry 2001; 44(4):273-294.

15. Dickinson ME, Simbuerger E, Zimmermann B, Waters CW, Fraser SE. Multiphoton excitation spectra in biological samples. J Biomed Opt 2003; 8(3):329-338.

16. Gurskaya NG, Fradkov AF, Pounkova NI, Staroverov DB, Bulina ME, Yanushevich YG, et al. A colourless green fluorescent protein homologue from the non-fluorescent hydromedusa Aequorea coerulescens and its fluorescent mutants. Biochem J 2003; 373(Pt 2):403-408.

17. Chudakov DM, Verkhusha VV, Staroverov DB, Souslova EA, Lukyanov $S$, Lukyanov KA. Photoswitchable cyan fluorescent protein for protein tracking. Nat Biotechnol 2004; 22(11):1435-1439.
18. Xia J, Lee DH, Taylor J, Vandelft M, Truant R. Huntingtin contains a highly conserved nuclear export signal. Hum Mol Genet 2003; 12(12):1393-1403.

19. Irwin S, Vandelft M, Pinchev D, Howell JL, Graczyk $\mathrm{J}$, Orr HT, et al. RNA association and nucleocytoplasmic shuttling by ataxin-1. J Cell Sci 2005; 118(Pt 1):233-242.

20. Guo B, Pearce AG, Traulsen KE, Rintala AC, Lee H. Fluorescence produced by transfection reagents can be confused with green fluorescent proteins in mammalian cells. Biotechniques 2001; 31(2):314-316, 318, 320-321.

21. Howell JL, Truant R. Live-cell nucleocytoplasmic protein shuttle assay utilizing laser confocal microscopy and FRAP. Biotechniques 2002; 32(1):80$82,84,86-87$.

22. Misteli T, Caceres JF, Spector DL. The dynamics of a pre-mRNA splicing factor in living cells. Nature 1997; 387(6632):523-527.

23. Wang L, Jackson WC, Steinbach PA, Tsien RY. Evolution of new nonantibody proteins via iterative somatic hypermutation. Proc Natl Acad Sci USA 2004; 101(48):16745-16749. 